\title{
Spatial Prisoner's Dilemma and Laws of Imitation in Social Psychology
}

\author{
Carlos Pelta \\ Dept. of Basic Psychology II, Complutense University, Madrid, Spain
}

Email address:

pelta.carlos@gmail.com (C. Pelta)

\section{To cite this article:}

Carlos Pelta. Spatial Prisoner's Dilemma and Laws of Imitation in Social Psychology, Social Sciences. Vol. 2, No. 2, 2013 , pp. $53-57$. doi:10.11648/j.ss.2013.0202.15

\begin{abstract}
It is designed a game based on the spatial prisoner introducing the three laws of imitation defined by Tarde. The French author described (1) the law of close contact, (2) the law of imitation of superiors by inferiors and (3) the law of insertion. The first law is defined like a Conformist rule; the second law is defined like a Maximization rule and the third law is defined like a Fashion rule or, alternating, a Snob rule. The method used to design the simulation in Java language programming is based on interactive evolutionary game rules which are constantly updated and is inspired by the paradigm of Chavalarias' metamimetic games. These games introduce very suggestive notions of Nonlinear Dynamics such as mimetic attractor or mimetic equilibrium. It is obtained the formation of little "clusters" of cooperators supporting these laws of imitation.
\end{abstract}

Keywords: Social Psychology, Spatial prisoner's dilemma, Laws of imitation, Jean-Gabriel Tarde, Chavalarias

\section{Introduction}

Jean-Gabriel Tarde introduced a theory of imitation and suggestion [1] emphasizing the preponderance of social factors behind learning and crime. He thought of the learning and the deviance [2] as very similar to the origins of fashions. According to Tarde, the human being, in the degree that he is social, is essentially imitative and that imitation plays a role in societies analogous to that of heredity in organic life. Tarde says that a human invention, by which a new kind of imitation is started (...) stands in the same relation to social science as the birth of a new vegetal to biology [1].

All resemblances of social origin are the direct or indirect product of various forms of imitation: custom-imitation or fashion-imitation, obedience-imitation, education-imitation, naïve imitation, deliberate imitation, etc. Actually repetitions are multiplications or self-spreading contagious [1]. Tarde considers that in the beginning of societies, the art of the chipping flint, of making bows, of leavening bread, for instance, must have spread like a contagion. In fact, every invention or discovery, tends to expand in its social environment, an environment which itself tends to self-expansion. Society is imitation and imitation is a kind of somnambulism [1].

The tendency to imitate is a natural thing. This tendency precedes the first social act but varies according to the nature of existing habits of imitation. Imitations in which logic has no place or extra-logical, are a kind of obedience based credulity and docility: people obey for the same reason that they believe [1]. In the family, for instance, the correlation between imitation and obedience is very clear. Tarde mentions as the father is the sovereign ruler of his child [1].

Imitation proceeds from the inner to the outer and in this way imitation passes on from one people to another, as well as from one class to another within the same people. Following Tarde the strongest proof that imitation spreads from within to without is that in the relations between different classes, envy never precedes obedience and trust but is always the result of a previous state of obedience and trust.

The socially learned acquisition is governed by what Tarde called the "three laws of imitation" [1], including (1) the law of close contact, (2) the law of imitation of superiors by inferiors and (3) the law of insertion.

The first law or law of close contact describes how individuals in close intimate contact with one another imitate each other's behaviour; that is, people have a greater tendency to imitate the customs and fashions of those with whom they have the most contact. Thus, people surrounded by people involved in a particular lifestyle is more likely to imitate these people than they would others with whom they had little association. Customs reflect long-term behaviour 
and fashions reflect short-term behaviour, and as population became denser behaviour would be oriented more toward fashion than toward custom.

Akers and Burgess in their Differential Reinforcement Theory [3] introduce Tarde's close contact law saying people learn to evaluate their own behaviour through interaction with significant others and groups in their lives. These groups not only control sources and reinforcements but also provide behaviours for observational learning. Also the deviant behaviour, originated by imitation, is sustained by social support from minority groups defining their behaviour as justified, rather than as undesirable.

The law of imitation of superiors by inferiors establishes people follow the model of high-status in hopes their imitative behaviour will get the rewards associated with being of a "superior" class [2]. Tarde [1] points that the fact that imitation proceeds from the inside to the outside of the model, it has to consist in a descent from the superior to the inferior. Combining the first and the second law, Tarde affirms that the things are most imitated are the most superior ones of those that are nearest [1].

The presumption of superiority from which the example emanates, attaches not only to the persons or classes, but to the time of its origin as well. Imitation is engaged in the currents of fashion and custom [1].

Tarde's third law is the law of insertion: new acts and behaviours are superimposed on old ones and subsequently either reinforce or discourage previous customs [2]. Insertion is correlative to invention. A new invention will eventually destroy the old invention to which it attaches itself. But when two mutually exclusive ways of doing something come into conflict, Tarde believed the newer one would ordinarily win out [2].

Game theory and, in particular, spatial prisoner [4], is an excellent testbed to analyze very relevant issues of Social Psychology: think of agents defined by a series of traits (behaviour, status, prestige...) and embedded in a social network where they can see some of the features of those with whom they interact. Agents can categorize the features of other agents in their environment. Imitation happens when an agent decides to take a trait that it has observed in one of its neighbours. Imitation rules present as input elements of an agent and its environment and as output a modifiable trait (a trait that an agent can change "voluntarily"); the agent will attempt to copy the trait of one of its neighbours. Such rules can be seen as part of the strategy of the agent and become modifiable traits.

It is defined an agent as an n-tuple trait $\rho$ taken from a multi-dimensional set of traits $\mathrm{T}$. These traits will be allowed to be divided into two categories: modifiable (M) and not entirely dependent on the willingness of the agent $(\mathrm{N})$. The traits of type (M) are those that an agent can change at will. The traits of type $(\mathrm{N})$ can become immutable, often dependent on global dynamics and change over long periods of time. For a given agent $\mathrm{A}$, the neighbourhood $\mathrm{A}$ is defined as the set of all agents that A can learn some traits from.

Imitation occurs when an agent decides to adopt a trait that it has observed in one of its neighbours. Mimetic games constitute a fascinating example of designing autoconfigurable computational simulations that use concepts based on the theory of Complex Systems. The peculiar characteristic of such games is handling mimetic rules, that is, imitation rules that constantly update themselves. From the viewpoint of Nonlinear Dynamics, these games introduce very suggestive notions such as mimetic attractor or mimetic equilibrium. An overview of imitation in line with the idea of imitation in Tarde [1] will be raised: it would be a conscious process by which the agent $\mathrm{A}$ would imitate $\mathrm{B}$ depending on an interdependence with respect to B's criteria, for example, because B is presented to A as more successful in terms of some criteria. In applying this aspect, imitation rules can be seen as part of the strategy of the agent and become modifiable traits. Thus, it is feasible to consider dynamics in populations of agents, described from imitation rule chains and defining a total reflexive order on the set of rules. Reflexivity appears because the imitation rules can update themselves. Reflexivity and imitation are two basic aspects in Neuroscience and Social Learning today. As the reader will easily understand, to include the reflexivity to model social systems, accommodates the fact that people quite often know that we use rules to make decisions and that we can monitor its use. But agents not only imitate according to their preferences but also form their preferences by imitation.

From a formal point of view, an imitation rule is defined like this:

Given an agent $\mathrm{A}$ and its neighbourhood $\mathrm{A}$, an imitation rule is a process that (a) assigns a value $v(\mathrm{~B}, \mathrm{~A})$, belonging to the set of the real numbers, to each agent $\mathrm{B}$ in $\mathrm{A}$ ( $V$ will be a valuation function) and (b) selects some traits that should be copied from the best agents (according to the values given in (a)) and defines the copying process [5].

The prisoner's dilemma [6]-[11] presents two players who can cooperate (C) or commit defection (D). If both cooperate they receive $R$ as payoff and $P$ in the case that they defect each other. In the event that their strategies are different, the one which cooperates receives a payoff $S$ and that who defects receives a payoff $T$. The payoff matrix is as follows: $T>R>P>S$. To carry out defection is always more advantageous from an individual perspective, but, collectively, mutual cooperation is the best thing you can do $(T+\mathrm{S}<2 \cdot R)$. The dilemma is that if the "rational" behaviour has to be based on maximizing the profits, the "rational" on the behalf of the agents is to behave carrying out defection. Yet, mutual cooperation enables higher performance than mutual disappointment to which is what the players seem doomed to again and again following the selfish strategy.

Consider two agents A and B with no memory. Each agent is in the neighbourhood of another and can choose between the action to cooperate (C) and the one to defect (D). In turn, and as regards their imitative behaviour, it can choose between a behaviour of imitation of neighbours which obtain higher payoffs (maximizing behaviour) or a conformist behaviour (imitation of the behaviour of the majority be- 
tween neighbouring agents). Behaviour (D) always brings higher payoffs than the behaviour $(\mathrm{C})$, the game is played repeatedly and agents change their strategy simultaneously according to its own rules. The definition of the rules is as follows:

-Maxi: if the neighbour agent gets higher payoffs, it copies its rule and uses it, updating its behaviour.

-Conf: if your strategy is different from that of the neighbouring agent, copy the rule and use it to change your behaviour, updating it.

According to Chavalarias [12] the activity of an agent consists in examining the consistency of its rules. For imitation rules $\left(r_{0}, r_{1}, \ldots, r_{n}\right)$, the question now is whether the strategy $\left(r_{0}, r_{1}, \ldots, r_{n}\right)$ is the best from the point of view of the agent. Otherwise, the agent will try to change its strategy, seeking a better one: or, some elements in $\left(\mathrm{r}_{0}, \mathrm{r}_{1}, \ldots, \mathrm{r}_{\mathrm{n}}\right)$ will be modified, yet remaining the initial rule $r_{1}$ as part of the strategy or $r_{n}$ will be changed.

Let's see how Chavalarias exposes this peculiar type of dynamics very different to the traditional mimetic dynamics. Following Chavalarias [12, p. 11] the state of game is given for the behaviour and the rule of imitation of each agent. Thus, for example, and for " $\mathrm{s}$ " expressing that state, $\mathrm{s}=\left[\mathrm{s}_{\mathrm{A}}\right.$ : (C, Maxi); $\left.\mathrm{s}_{\mathrm{B}}:(\mathrm{D}, \mathrm{Conf})\right]$. In this simple game there is only 16 possible states. For example, if the initial state is $\mathrm{s}=\left[\mathrm{s}_{\mathrm{A}}:(\mathrm{C}\right.$, Maxi); $\left.\mathrm{s}_{\mathrm{B}}:(\mathrm{D}, \mathrm{Conf})\right]$, after a period, A will become a conformist agent because its neighbour $\mathrm{B}$ has been the most successful and, in turn, will change its behaviour (C) for behaviour (D), in order to follow the behaviour of B. In contrast, agent B will take the Maxi behaviour to resemble the agent $\mathrm{A}$ and will continue to play (D), since this is the most profitable type of action. In a second round, both agents will have the same behaviour and, consequently, the same payoffs. Only the agent A will change its strategy to be like agent $B$ and they will both end with the strategy (D, Maxi). The final state of the game will be $\mathrm{s}^{\prime \prime}=[(\mathrm{D}$, Maxi $) ;(\mathrm{D}$, Maxi) $]$; $\mathrm{s}^{\prime \prime}$ will be reachable from the states $\mathrm{s}=[(\mathrm{C}$, Maxi);(D, Conf) $]$ and s'=[(D, Conf);(D, Maxi) $]$. More generally, an s' state is reachable from an s state, if and only if a system that starts in s state can reach the s' state in a finite number of mimetic transitions [5]. See the Fig. 1:

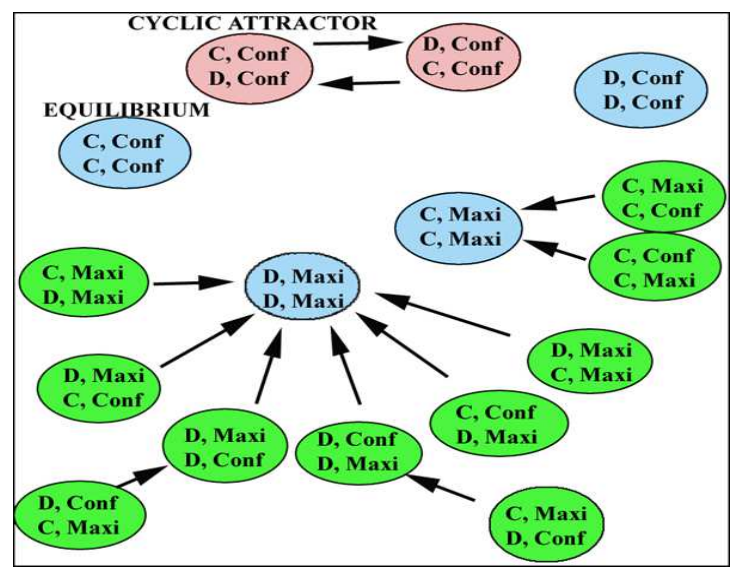

Figure 1. Metamimetic dynamics by Chavalarias [12, p. 11].

\section{Method}

It is designed a game based on the spatial prisoner which simulates Tarde's three laws of imitation above described.

Our simulation has been programmed in JAVA J2SE 5.0 programming language and presents the following characteristics:

(a) There is a lattice of $\mathrm{n} x \mathrm{n}$ cells, occupied by an $\mathrm{N}$ initial population of 1,000 individuals (obviously, $\mathrm{n}$ and $\mathrm{N}$ are adjustable parameters). The payoffs for the prisoner's dilemma matrix are the values $R=1, T=b(b>1), S=P=0$.

(b) $1<b<2$; the initial distribution of cooperators takes their values from the set $\{0.1,0.2,0.3,0.4,0.5,0.6,0.7,0.8$, 0.9 .

(c) The neighbourhood of each individual is composed of the 8 adjacent cells plus the the individual's own cell, supporting Tarde's law of close contact (LCC).

(d) The agent plays with its 8 neighbours and itself.

(e) The new payoffs of the agents are computed by adding the 9 results of a game between 2 players.

(f) The following imitation rules are introduced: (f1) Conf rule or Conformist rule simulating the law of close contact (LCC); if your behaviour is different from that of the neighbouring agent, copy its behaviour; (f2) Maxi rule or Maximization rule simulates the law of imitation of superiors by inferiors (LIS) and is so defined: if the neighbour agent gets higher payoffs, copy its behaviour; (f3) Fashion rule: copy the behaviour with the highest frequency of appearance in your neighbourhood (in case of equal frequency, copy at random); (f4) Snob rule: copy the behaviour with a lower frequency appearance in your neighbourhood (if the frequency of behaviour appearance is the same, copy at random).

(f3) and (f4) simulate the law of insertion (LOI), alternating the copy of the latest choice made with the Fashion rule and the copy using the Snob rule in every round of the game. The agents have memory for these two rules for the 3 previous rounds of the game.

(g) In each period and for each agent, each imitation rule can be updated itself. For instance, if an agent A adopted the imitation rule (f1) and if the majority of its neighbours moved to the rule (f2) in the last round of the game, then the agent $\mathrm{A}$ will adopt the rule (f2). By referring rules to other rules, a mimetic endogenous dynamics is reflected, in which the rules interact, constantly updated reflectively. For this reason, unlike the programming of classical evolutionary game rules, based on replicator dynamics [13], [14],[15],[16] our mimetic dynamics is not exogenous, that is, it is not imposed by rigid rules externally fixed by the programmer and unable to evolve according to its encounter with other rules.

(h) Every imitation rule (possibly new) will be used to update the behaviour of the agent A. If the imitation rule of $A$ is (fl) and A cooperated in the final round, but one of its neighbours $\mathrm{B}$ which committed defection, obtained in the last round, higher payoff than A and the rest of its neighbours, the agent $\mathrm{A}$ will become a defection practicing agent 
in the next round.

\section{Results}

Once taken into account all these features of our computational simulation, the results are analyzed.

For $b=1.4$, an initial rate of 0.5 cooperators, 1,000 agents, 100 rounds of the game, and a memory $M$ of the 3 previous rounds in the case of rules (f3) and (f4), it is obtained the following Fig. 2:

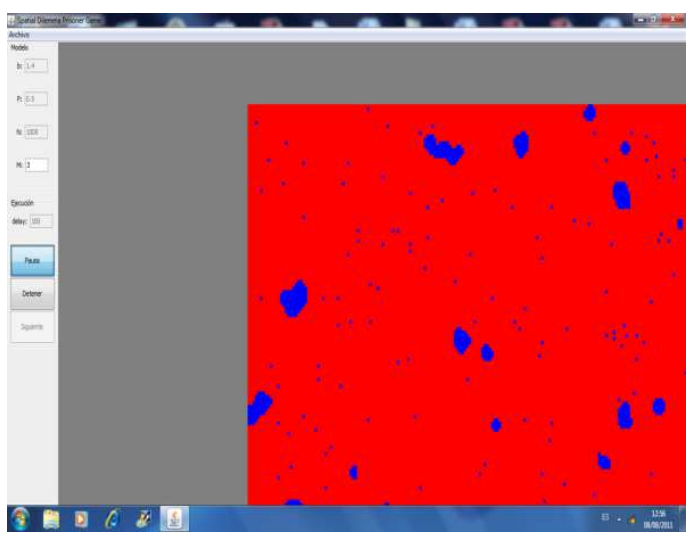

Figure 2. Spatial prisoner's dilemma for $b=1.4$.

For a uniform initial distribution of rules and actions, the system is in static equilibrium in round $60 ; 15$ small stable "clusters" and 1 "blinker" have formed of cooperator agents (in blue) and, although initially half of the population consists of cooperating agents, in the end, they only reach $35 \%$ of the total. For example, if it is compared this result with those obtained in cases in which $b=1.6$ and $b=1.9$ and for an initial rate of 0.5 and always a memory of the 3 previous rounds, it is possible to find that the patterns are very similar (as shown in Fig. 2 and Fig. 3). The similarity of $b=1.6$ with the previous pattern is apparent. There is only one attractor, being in equilibrium in round 50, no oscillators are generated and the distribution of cooperating agents is also concentrated in only 5 "clusters", existing a wide dispersion of these agents across the array. Its percentage reaches near $28 \%$, given the lower payoff to defectors. See the Fig. 3:

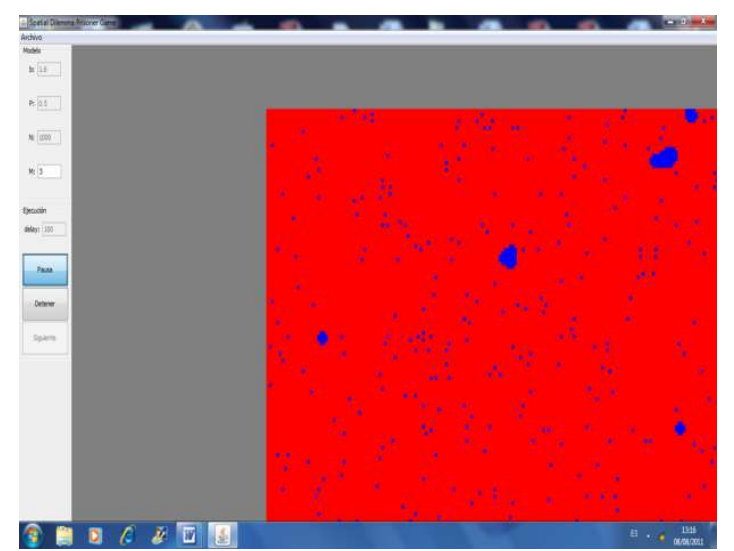

Figure 3. Spatial prisoner's dilemma for $b=1.6$.
If $b=1.9$ and keeping all other parameters the same, it is again found a similar pattern, but now the static equilibrium is reached in round 41 . There is only 1 "cluster" formed by a few agents. Without doubt, this is an evolutionary form of survival against a tide of defectors which are paid very high for it. It seems as if among the cooperators prevails direct reciprocity as a way to respond to the invaders which practice defection. The proportion of cooperating agents is slightly above $20 \%$. As expressed in the final section of conclusions, it can be said that cooperation has been largely defeated by the defection. This result will be explained appealing to the effect of Tarde's two last imitation laws. See the Fig. 4:

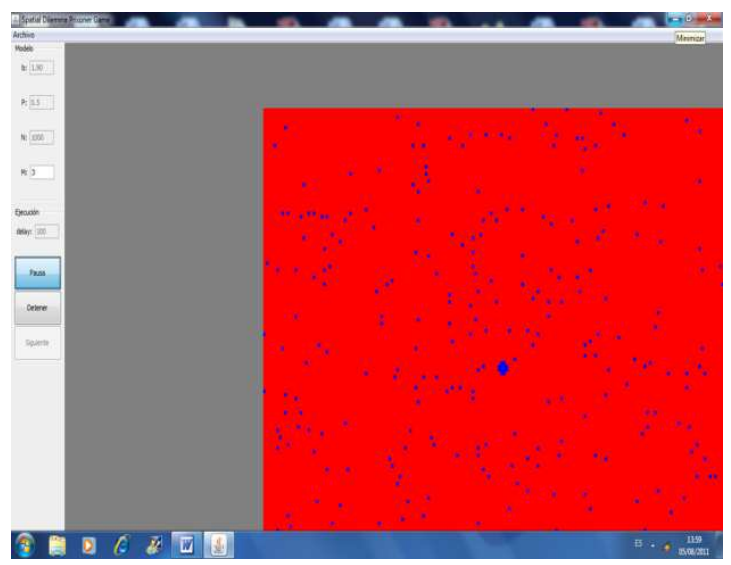

Figure 4. Spatial prisoner's dilemma for $b=1.9$..

It has been conducted a comprehensive tour of all the possibilities, combining all possible values of $b$ between 1.1 and 1.9, with an initial distribution of cooperators between 0.1 to 0.9 and a memory $M$ between 1 and 9 rounds for the rules (f3) and (f4). Changing the population of agents and increasing the number of rounds to 100 , the results are qualitatively and quantitatively very similar to those outlined above. It is necessary try to explain the meaning of these results.

\section{Discussion}

It has been introduced a special type of dynamics based on the spatial prisoner's dilemma interacting with imitation rules which simulate the behaviour of Tarde' simitation laws: (1) close contact (LCC); (2) imitation of superiors by inferiors (LIS) and (3) insertion (LOI). In this work it has been showed that in this game the imitation rules yield a great power and preferential attractor. In fact, the most original aspect of our work has been to mix purely stochastic imitation rules ( $\mathrm{f} 3$ and $\mathrm{f} 4$ ) with non stochastic classical rules (f1 and $\mathrm{f} 2$ ). And yet it has been impossible to relax the dynamics: the equilibrium is static.

Even bigger the surprise, however, the low proportion of cooperating individuals registered in our successive simulations. With a similar approach, Chavalarias and Bourgine [5] obtain about a $5 \%$ of cooperators for $b=2.5$ and only an initial $10 \%$ of cooperators, which was predictable. However, 
they reach up to $83 \%$ of the cooperators for $b=1.2$ and, of course, an initial $90 \%$ of them. Even with initial ratios of $90 \%$ of cooperators, and varying other parameters together, find the formation of large and numerous "clusters" of defectors which, at worst, become easily to occupy $30 \%$ of the total population at the end of the simulation. Question is raised about the drag on the development of cooperation that could involve the introduction of the four rules of imitation fixed. Low population proportion of cooperators for all types of payoffs that defectors can receive, has an explanation. Although two rules of stochastic nature have been introduced, its effect is nullified by the very mimetic dynamics, which means that they can not even be present in the attractor. Thus, agents attracted by non stochastic rules, and $b$ values that increasingly are encouraging defection, are mass defined as defectors which find ways to maintain their payoffs as high as possible. But this circumstance supports Tarde's law of imitation of superiors by inferiors (LSI) because the imitation of the agents with higher payoffs (defectors) is majority also including the case with an initial rate of 0.9 cooperators receiving a payoff of 1 (defectors receive payoffs from 1.1 to 1.9). Besides our simulation verifies Tarde's law of insertion (LOI) combining rules (f1) and (f2) because the most imitated behaviour or Maximization behaviour makes, via Conformist rule or rule (f1), the new behaviour reinforced, discouraging the cooperative behaviour of the agents with lesser payoffs.

\section{References}

[1] G. Tarde, The Laws of Imitation. New York: Holt, 1903.

[2] G. Williams, "Gabriel Tarde and the imitation of deviance". Available: http://soc506-ni.blogspot.com/2009/03/tarde-and-imitationof-deviance.html.
[3] R.L. Ackers and R.L. Burgess, "A differential association-reinforcement theory of criminal behavior", Social Problems, vol. 14, pp. 128-147, 1966.

[4] M. Nowak and R. May, "Evolutionary games and spatial chaos”, Nature, 359, pp. 826-829, 1992.

[5] D. Chavalarias and P. Bourgine, "Metamimetics and the spatial prisoner's dilemma". Available: nlin.AO70301005.

[6] R. Axelrod, The Evolution of Cooperation. New York: Basic Books, 1984.

[7] D.R. Gilbert, "The prisoner's dilemma and the prisoners of the prisoner's dilemma", Business Ethics Quarterly, 6, pp. 165-178, 1996.

[8] D. Gordon, "Is the prisoner's dilemma an insoluble problem", Mind, 93, pp. 98-100, 1984.

[9] I. Haji, "Escaping or avoiding the prisoner's dilemma", Dialogue, 30, pp. 153-162, 1991.

[10] W. Poundstone, Prisoner's Dilemma. New York: Doubleday, 1992.

[11] P. Grim, "Undecidability in the spatialized prisoner's dilemma", Theory and Decision, 42, pp. 53-80, 1997.

[12] D. Chavalarias, "Metamimetic games: modeling metadynamics in social cognition", JASSS, vol. 9, http://jasss.soc.surrey.ac.uk/9/2/5.html.

[13] J.W. Weibull, Evolutionary Game Theory. Mass.: MIT Press, 1995.

[14] J. Hofbauer and K. Sigmund, Evolutionary Games and Replicator Dynamics. Cambridge:CUP, 1998.

[15] H. Gintis, "Classical versus evolutionary game theory", Journal of Consciousness, vol. 7, pp. 300-304, 2000.

[16] G. Mar, "Evolutionary game theory, morality, and darwinism”, Journal of Consciousness Studies, vol. 7, pp. 322-326, 2000 . 